\title{
DIC MODEL ON MICE. TISSUE FACTOR OR PLATELET AGGREGATION INDUCERS?
}

\author{
Linara I. Bashirova
}

Izhevsk State Medical Academy

\begin{abstract}
The purpose of this study is to evaluate the validity of the classical model of DIC-syndrome in the conditions of tissue factor initiation. The study was performed on 60 male mice. All laboratory animals were divided into two groups: the first group of mice used a mixture of solutions of $0.5 \mathrm{mg} / \mathrm{kg}$ of collagen and $0.06 \mathrm{mg} / \mathrm{kg}$ of epinephrine as a trigger for thrombosis, the second group - $10 \mathrm{mg} / \mathrm{kg}$ of recombinant tissue factor. In conditions of systemic thrombosis, injected suspension of epinephrine and collagen (group I) resulted in the death of all animals within 3 days. Notably, more than $50 \%$ of the mice died within the first hour of the experiment. The death rate in group II on the 14th day of the experiment was $100 \%$, but in contrast to group I, the death of laboratory animals was registered daily throughout the entire observation period $(2.0 \pm 1.8$ vs $133.5 \pm 124.7, p<0.01)$. The results show uniform loss in laboratory animals after induction of thrombosis, and morphological evaluation of animals is characterized by a low content of blood clots in the lungs of lab animals, suggesting the root cause of death of animals is not in acute thrombosis but in the development of delayed manifestations of DIC, which this model does not presuppose since it is designed to evaluate the effectiveness of new antiplatelet drugs in the acute experiment. Thus, the findings demonstrate the limitations of the use of tissue factor in model thrombosis and justify the feasibility of using physiological aggregation inducers.
\end{abstract}

Key words: model of DIC-syndrome, tissue factor, agonists of aggregation. 


\section{INTRODUCTION}

Disseminated blood clotting (DIC) is a syndrome characterized by pathological disregulation of hemostatic and fibrinolytic processes (Bick., 2002), which, in fact, is only a complication of the underlying disease, but often determines the severity of the disease and the fatal outcome (Levi et al., 1999). To investigate the main pathophysiological mechanisms that will allow developing effective therapy for patients with DIC-syndrome, appropriate models of disseminated intravascular coagulation in animals were developed using various animal species, a wide range of DIC inducers, their routes of administration and the duration of inductions. One of the most common models of DIC is the model proposed by DiMinno (DiMinno and Silver., 1983). The analysis of publication activity reveals a fairly wide use of this model by researchers (Gurevich et al., 2018, Urakov et al., 2017). The classic version is intravenous injection of platelet aggregation inducers into the tail vein of a mouse. However, recently there have been suggestions to modify methods for modeling DIC-syndrome - replacing the initiating agent-aggregation inducers (a mixture of epinephrine and adenosine diphosphate) with a more pathophysiological tissue factor. In this regard, the purpose of this study is to evaluate the validity of the classical DIMinno model in the conditions of DIC initiation by a tissue factor and to compare it with the classical version.
Experimental research in vivo is performed on 60 viripotent lab rats in compliance with the International

recommendations of the European Convention on protection of vertebrate animals for experimental animals, laboratory practice regulations upon conducting pre-clinical studies in Russia (GOST 3 51000.3-96 and 51000.4-96, GOSTR 50258-92)) and the order of the Ministry of Health and Social development of Russia № 708n dated from 23/08/2010 "On approval of the rules for laboratory practice" (GLP). The research was approved by the ethics Committee of the lzhevsk state medical Academy (Protocol No. 2 dated 20.12.2018).

Modeling of generalized thrombosis was performed by introducing aggregation inducers into the tail vein of mice. All the laboratory animals were divided into two groups. In the first group of mice, a mixture of solutions of $0.5 \mathrm{mg} / \mathrm{kg}$ of collagen and $0.06 \mathrm{mg} / \mathrm{kg}$ of epinephrine was used as a trigger for thrombosis ('Technology-standard", Russia), in the second group - $10 \mathrm{mg} / \mathrm{kg}$ of recombinant tissue factor (Innovin ${ }^{\circledR}$, Dade Behring, Germany). The animals were observed for 14 days. After the animals were withdrawn from the experiment, the models were compared according to the following indicators: survival-death rate, results of macroscopic evaluation of mouse lungs, and histological examination of the lungs.

Materials and methods.

2021 January Edition | www.jbino.com | Innovative Association 
To perform light microscopy the material was fixed in $10 \%$ formalin solution. The standard histologic preparation was followed by paraffin sections with microtome LEICA RM 2145 (Leica Biosystems, Germany) which were stained with hematoxiline-eosin. Further, morphological changes were evaluated in 10 vision fields using Leica CME binocular microscope (Leica Biosystems, Germany) at the light optical level at $\times 40$ and $\times 100$ magnification.

The findings are processed using the statistical package Statistica 10.0 (StatSoft Inc, USA). The normality of the distribution of actual data was checked by using the criterion of Shapiro-Wilka. Survival analysis in DIC-syndrome modeling was performed using the Kaplan-Meyer method. Differences in survival between groups were evaluated using the Wilcoxon test. Critical level of $p$ significance for statistical criteria was taken equal to 0.05 .

\section{Results.}

The results of the survival assessment of laboratory animals depending on the initiating factor of DIC-syndrome are presented in table 1.

In conditions of systemic thrombosis, injected suspension of epinephrine and collagen (group I) resulted in the death of all animals within 3 days. Notably, more than $50 \%$ of group I mice died within the first hour of the experiment. Macroscopically, the lung volume is increased. Multiple blood clots in the form of "wormlings" are found on the incisions. Microscopically, the pattern of the lungs is heterogeneous. The parenchyma of the lungs is edematous, the vessels are fullblooded, there are foci of Iymphohistocytic infiltration with an admixture of neutrophils. In the arterial vessels of the lobe arteries, one can detect blood clots of a mixed nature, completely or partially obstructing the lumen (figure 1B).

The death rate in group II on the 14th day of the experiment was $100 \%$, but in contrast to group I, the death of laboratory animals was registered daily throughout the entire observation period. It is interesting that the macroscopic assessment of the extracted lungs shows a significant decrease in the area of thromboembolic lesion, but at the same time the arterial vessels of small and medium calibers show mixed blood clots, completely or partially obturating the lumen (figure 1C).

Analysis of the literature data demonstrates the increased interest of a number of researchers in TF as the initiator of thrombosis in simulative states. The main arguments for the use of tissue factor are, on the one hand, pathogenicity, on the other hand comprehensive information on the action of tissue factor both in vitro and in vivo (Nemerson., 1988.). Different researchers suggest using recombinant TF extracted from rabbit brain (Warr et al., 1990, Asakura et al., 2001, Mohri et al., 1997) or lung tissue (Tunali et al., 2004, Mischke et al., 2005). This mixture of phospholipids and TF is often referred to as thromboplastin. However, here, in my opinion, there are a number of problems that potentially limit the use of TF in experiments.

The use of self-made thromboplastin makes it impossible to standardize the content of tissue factor, in contrast to commercial sets of recombinant tissue factor. And it would seem that the solution to this problem is possible via using a ready-made commercial 
reagent. However, according to Olrik B. L. et al. thromboplastin, which is produced in self-own laboratories and even purchased as a ready-made reagent, is very often heavily contaminated with endotoxin. And endotoxin is a strong initiator of blood clotting and triggers DIC in sepsis, which means it can affect the purity of the experiment and the reproducibility of the results obtained.

The own findings show uniform loss in lab animals after initiation of thrombosis, and morphological evaluation of animals is characterized by a low content of blood clots in the lungs of lab animals, suggesting the root cause of death of animals is not in acute thrombosis but in the development of delayed manifestations of DIC, which this model does not presuppose since it is designed to evaluate the effectiveness of new antiplatelet drugs in the acute experiment. In the era of receptorassociated pharmacology and therapy, we need more subtle means to evaluate the effectiveness of pharmaceutical drugs depending on their mechanism of action, which keeps the relevance of using physiological inducers of platelet aggregation, but under conditions of the whole organism. It is the different ways of platelet activation depending on the nature of the inducer that allows us to assume or confirm the mechanism of the anti aggregational effect of a potential drug and to ensure continuity of the transition from the research stage in vitro to laboratory animals.

Table 1: Survival rates of mice in the simulation of systemic collagen-epinephrine thrombosis, $\mathrm{M} \pm \mathrm{SD}$

\begin{tabular}{|l|c|c|}
\hline Group of animals & Group I & Group II \\
\hline Life time, hours & $2,0 \pm 1,8$ & $133,5 \pm 124,7 *$ \\
\hline Note $-*$ the level of statistical significance of differences between the control and experimental \\
groups $\mathrm{p}<0.01$ at $\chi 2=33.1$
\end{tabular}



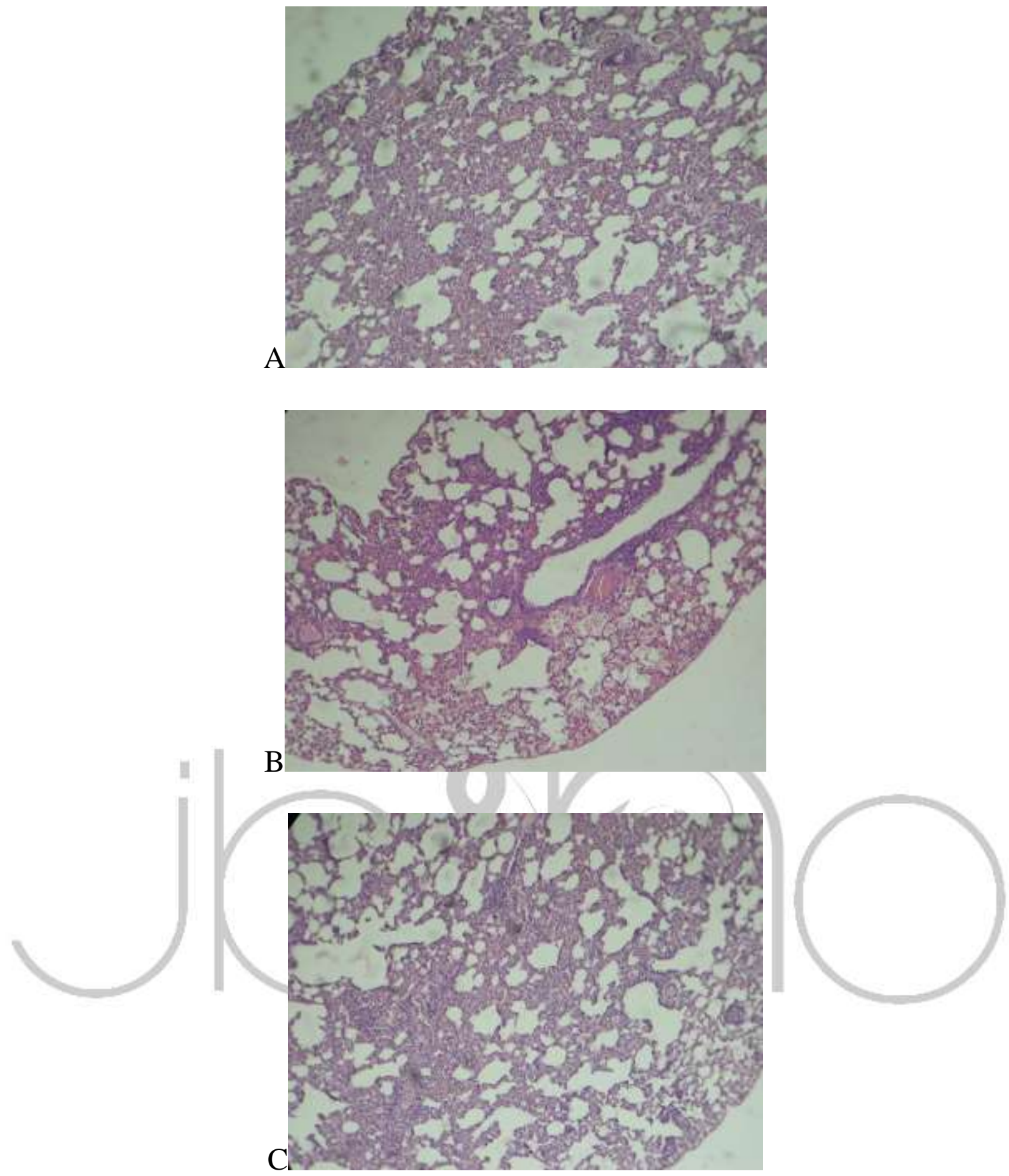

Figure 1 - Example of a histological pattern of mouse lungs (hematoxylin and eosin staining, X100). Ahealthy mouse, B-Group I, C-Group II. 
Conclusion.

Thus, based on the results of the study, the example of the classical model of DiMinno, demonstrates the limitations of the use of tissue factor as an initiator of thrombosis in animals and justifies the feasibility to use physiological inducers of aggregation.

\section{Acknowledgements.}

No

\section{References.}

Bick, R.L. 2002. Disseminated intravascular coagulation: a review of etiology, pathophysiology, diagnosis, and management: guidelines for care. Clin Appl Thromb Hemost.8(1):1-31.

Levi, M., De, J.E., Van der, P.T. 1999. Disseminated intravascular coagulation. Thromb Haemost. 82(2):695-705.

DiMinno, G. and Silver, M. J. 1983. Mouse antithrombotic assay: a simple method for the evaluation of antithrombotic agents in vivo. Potentiation of antithrombotic activity by ethyl alcohol. Journal of Pharmacology and Experimental Therapeutics. 225(1):57-60.

Gurevich, K.G., Urakov, A.L, Bashirova, L.I., Samorodov, A.V., Purygin, P.P., Yermokhin, V.A., Gilmutdinova, A.S. and Bondareva, N.A. 2018. The hemostatic activity of bis (2-aminoethan-1-sulfonate) calcium. Asian Journal of Pharmaceutical and Clinical Research. 11 (11):452-455.

Urakov, A. L, Samorodov, A.V., Kamilov, F.K., Khaliullin, F.A., Khalimov, A.R. 2017. Dynamics of thrombosis and hemostasis system indicators in rats with thrombosis of inferior vena cava in experiment as a model for preclinical studies. Biomed Pharmacol J. 10(1):237-245.

Urakov, A.L., Samorodov, A.V., Kamilov, F.Kh., Khaliullin F.A., Gubaeva, R.A. 2017. Hemostatical activity of new benzylammonium salt, 2-[3-methyl-1-npropyl-7-(1,1- dioxotiethanyl-3)xantinyl-8thio]acetic acid. Natl J Physiol Pharm Pharmacol 2017;7(11):1213-1218

Nemerson, Y. 1988. Tissue factor and hemostasis. Blood.71(1):1-8.

Warr, T.A., Rao, L.V. and Rapaport S.I. 1990. Disseminated intravascular coagulation in rabbits induced by administration of endotoxin or tissue factor: effect of anti-tissue factor antibodies and measurement of plasma extrinsic pathway inhibitor activity. Blood. 75(7): 1481-9.

Asakura, H., Aoshima, K. and Ichino, T. 2001. All-trans retinoic acid is partially effective against lipopolysaccharideinduced but not against tissue-factorinduced disseminated intravascular coagulation in rat models. Blood Coagul Fibrinolysis. 12(4):301-6.

Mohri, M., Gonda, Y. and Oka M. 1997. The antithrombotic effects of recombinant human soluble thrombomodulin (rhsTM) on tissue factorinduced disseminated intravascular coagulation in crab-eating monkeys (Macaca fascicularis). Blood Coagul Fibrinolysis. 8(5):274-83.

Tunali, T., Yarat, A., Bulut, M. 2004. 6,7Dihydroxy-3-phenylcoumarin inhibits thromboplastin induced disseminated 
intravascular coagulation. $\mathrm{Br} \mathrm{J}$ Haematol. 126(2):226-30.

Mischke, R., Fehr, M., Nolte, I. 2005. Efficacy of low molecular weight heparin in a canine model of thromboplastininduced acute disseminated intravascular coagulation. Res Vet Sci. 79(1):69-76.

Olrik BL, Thuri KA, Wiinberg B. 2009. Implementation of the ISTH classification of vnon-overt DIC in a thromboplastin induced rabbit model. Thromb Res. 124(4):490-7. 